\title{
Coupled THINC and level set method: a conservative interface capturing scheme with arbitrary-order surface representations
}

\author{
Longgen Qian ${ }^{\mathrm{a}, \mathrm{b}}$, Yanhong Wei ${ }^{\mathrm{a}, *}$, Feng Xiao ${ }^{\mathrm{b}, *}$ \\ ${ }^{a}$ College of Materials Science and Technology, Nanjing University of Aeronautics and Astronautics, Nanjing, 211100, China \\ ${ }^{b}$ Department of Mechanical Engineering, Tokyo Institute of Technology,4259 Nagatsuta Midori-ku, Yokohama, 226-8502, Japan.
}

\begin{abstract}
In this paper, we propose a simple and accurate numerical method for capturing moving interfaces on fixed Eulerian grids by coupling the Tangent of Hyperbola Interface Capturing (THINC) method and Level Set (LS) method. The innovative and practically-significant aspects of the proposed method, so-called THINC/LS method, lie in (1) representing the interface with polynomial of arbitrary-order rather than the plane representation commonly used in the Piecewise Linear Interface Calculation (PLIC) Volume of fluid (VOF) methods, (2) conserving rigorously the mass of the transported VOF field, and (3) providing a straightforward and easy-to-code algorithm for implementation. We verified the proposed scheme with the widely used benchmark tests. Numerical results show that this new method can obtain arbitrary-order accuracy for interface reconstruction and can produce more accurate results than the classical VOF methods.
\end{abstract}

Keywords: Moving interface, multiphase flow, VOF, THINC, level set, interface reconstruction, surface representation.

\section{Introduction}

Moving interface phenomena, such as multiphase flows, moving fronts with chemical reactions or phase change, are widely found in nature and engineering fields. Numerical modeling and computation of moving interfaces have a long history of researches with large amount efforts devoted, but still remain a challenging and unresolved topic. Numerous methods have been proposed to identify and compute moving interfaces by using either marking points [1, 2, 3, 4] or identification functions. The latter approach solves the identification function for moving interface on fixed Eulerian grids, and is efficient and robust for interface which experiences even topological changes, like

${ }^{*}$ Corresponding author: Dr. Yanhong. Wei (Email: nuaadw@126.com); Dr. F. Xiao (Email: xiao.f.aa@m.titech.ac.jp) 
merging and breaking. The most popularly used identification functions are the volume fraction or volume of fluid (VOF) function [5, 6, 7, 8, 9, 10] and the level set (LS) function [11, 12, 13].

The idea behind the VOF methods is to use the volume fraction (VOF function) of the target fluid in each grid cell and identify the interface by the cells where the volume fraction values fall between 0 and 1 . The VOF methods update the VOF function by solving the advection equation with the finite volume scheme, which ensures the mass conservation of the VOF function. A class of schemes have been devised so far to explicitly reconstruct the interface with straight or curved lines (plane or curved surface in three dimensions) from the given volume fraction values. For example, in the original VOF scheme [5] and the earlier Simple Line Interface Calculation (SLIC) scheme [14], the interface is represented by a straight line parallel to the grid axis, which results in at best the first-order accuracy. As a more accurate algorithm, Piecewise Linear Interface Calculation (PLIC) [6, 15] algorithm was proposed where the orientation (the normal direction) of the cell-wise interface line segment is taken into account. To improve the accuracy of the orientation computation, the least squares volume-of-fluid interface reconstruction algorithm (LVIRA) [16] and the efficient least squares VOF interface reconstruction algorithm (ELVIRA) [10] were developed. Rather than the linear approximation in the SLIC and PLIC schemes, efforts to fit the interface with higher order surface representations have been also made in the past years, for instance, the least-square fit (LSF) [17, 18] algorithm, the spline interface reconstruction (SIR) [19] algorithm, piecewise-planar interface reconstruction with cubic-Bezier fit (CBIR) [20] algorithm, and the Quadratic Spline based Interface (QUASI) [21] algorithm. Although these algorithms show some improvements compared to the PLIC method, to our knowledge, all these algorithms are limited to 2D and none of them can realize interface reconstruction beyond second-order. The abovementioned VOF methods, known as the geometrical type VOF methods, explicitly use geometrical elements, such as line or surface segments cutting through grid cells, to formulate the interface, thus involve complicated algorithmic procedure which makes the following tasks formidably difficult in 3D case: (1) approximating interface with higher-order surface representation, and (2) implementing for three-dimensional cases.

In contrast to the geometrical VOF schemes mentioned above, the Tangent of Hyperbola Interface Capturing (THINC) [22, 23, 24, 25, 26, 27] schemes, use an implicit approach to embed the geometrical information in the THINC function, a regularized hyperbolic tangent function, which is Sigmod function mimicking the VOF function. Given the VOF values in all cells, the THINC function can be cell-wisely constructed as continuous function in each cell, which is then used to compute the numerical fluxes needed in the finite volume formulation to update the VOF function. The THINC scheme can be simply used with normal finite volume advection schemes to capture moving interfaces in practice. Some variants of THINC method have developed, including multi-dimensional THINC reconstruction in Cartesian grid [24] and unstructured grids in 2 and 3D [25, 27] with linear and quadratic surface rep- 
resentations. It is revealed that the schemes with multi-dimensional THINC reconstructions are able to give numerical results competitive to existing PLIC type VOF methods and show great practical significance and potential for further extension.

It should be noted that being a discontinuous (or steep-gradient) field function, the VOF function cannot provide accurate and reliable geometrical information for the interface, while the level set method show great superiority as discussed next.

Being another methodology for interface capturing, the level set methods have gained increasing popularity in recent years. Unlike the VOF methods, the level set methods employ the smooth signed distance function to represent the interface. The signed distance function, also known as the LS function, is a set of smooth hypersurfaces or manifolds of higher dimensions, which can be solved by high-order schemes for advection equation. Furthermore, given the signed distance function, the level set methods can provide adequate geometrical information of the interface, such as normal vector and curvature, needed in formulating physical models for interface and reconstructing the interface as explored later in this paper. During the evolution of moving interface the values of LS function in the computational domain may not remain always the distance to the interface, so the reinitialization process [13, 28] is needed in practice to modify the values of the level set function on grid cells. In principle, as the numerical conservativeness is not guaranteed in either advection (evolution) or reinitialization computation, mass loss or gain may occur to the fluid of interest, which is the fatal drawback of level set method. Several efforts have been made to remedy the conservation error for level set methods, for example, the coupled particle/level set (Particle LS) [29] and the coupled the level set/VOF methods (CLSVOF) [30, 31, 32, 33]. Unfortunately, these methods are of significant algorithmic complexity, and none of them realizes both numerical conservativeness and high-order interface representation.

In this paper, we present a novel interface capturing scheme, so-called THINC/LS (THINC scheme coupled with the LS method), which possesses the following major new properties in comparison with other state-of-the-art methods.

- Instead of the plane and quadratic surface representations used in the existing geometrical VOF methods, higherorder (arbitrary order in principle) surface polynomials can be determined from the level set function, and then used to build the THINC or VOF function.

- The interface in the level set field, i.e. the 0-level surface in the level set function, is synchronized with the interface identified from the THINC reconstruction with the mass conservation constraint. Thus, the numerical conservativeness is ensured.

- The function pair of THINC and level set are updated and coupled simultaneously through simple and straight- 
forward solution procedure without the complex computations involved in the geometrical VOF algorithms.

We have extensively examined the THINC/LS method regarding interface reconstruction and transport of moving interfaces. The numerical results verify the superiority of the method in comparison with other existing methods.

This paper is organized as follows. The details of the proposed THINC/LS scheme are described in section 2. Interface reconstruction tests are presented in section 3. Extensive numerical verifications with widely used benchmark tests are presented in section 4 . We end this paper with some conclusion remarks in section 5.

\section{Numerical method}

\subsection{The interface indication functions}

Although the idea presented in this work can be in principle extended to any structured and unstructured mesh, we limit our presentation to Cartesian grid. The computational domain is divided by mesh cell $\Omega_{i j k}=\left[x_{i-\frac{1}{2}}, x_{i+\frac{1}{2}}\right] \times$ $\left[y_{j-\frac{1}{2}}, y_{j+\frac{1}{2}}\right] \times\left[z_{k-\frac{1}{2}}, z_{k+\frac{1}{2}}\right]$, where $i, j$ and $k$ denote the cell index in $x, y$ and $z$ directions respectively. We also denote the six surface segments of $\Omega_{i j k}$ by

$$
\begin{aligned}
& S_{i \pm \frac{1}{2} j k}: x_{i \pm \frac{1}{2}} \times\left[y_{j-\frac{1}{2}}, y_{j+\frac{1}{2}}\right] \times\left[z_{k-\frac{1}{2}}, z_{k+\frac{1}{2}}\right], \\
& S_{i j \pm \frac{1}{2} k}:\left[x_{i-\frac{1}{2}}, x_{i+\frac{1}{2}}\right] \times y_{j \pm \frac{1}{2}} \times\left[z_{k-\frac{1}{2}}, z_{k+\frac{1}{2}}\right], \\
& S_{i j k \pm \frac{1}{2}}:\left[x_{i-\frac{1}{2}}, x_{i+\frac{1}{2}}\right] \times\left[y_{j-\frac{1}{2}}, y_{j+\frac{1}{2}}\right] \times z_{k \pm \frac{1}{2}} .
\end{aligned}
$$

In the present work, we use a mesh of uniform spacing $\left(\Delta x=x_{i+\frac{1}{2}}-x_{i-\frac{1}{2}}=\Delta y=y_{j+\frac{1}{2}},-y_{j-\frac{1}{2}}=\Delta z=z_{k+\frac{1}{2}}-z_{k-\frac{1}{2}}\right)$ for simplicity. The center of each cell locates at $\left(x_{i}, y_{j}, z_{k}\right)$.

Considering an interface $\partial V$ lying between two regions, $V^{1}$ and $V^{2}$ filled with different fluids in $\mathbf{R}^{2}$ or $\mathbf{R}^{3}$, we introduce two interface indicator functions, i.e. the level set (LS) function $\phi(\mathbf{x}, t)$ and the THINC function $H(\mathbf{x}, t)$, as illustrated in Figure 1 for the one-dimensional case.

- LS function $($ Figure 1 $(\mathrm{a}))$ :

The distance from a point $\mathbf{x}=(x, y, z)$ to the interface $\partial V$ is defined by

$$
\operatorname{dist}(\mathbf{x}, \partial V)=\inf _{\mathbf{x}_{l} \in \partial V}\left\|\mathbf{x}-\mathbf{x}_{I}\right\|
$$

where $\mathbf{x}_{I}$ represents any point on the interface, and is called the interface point hereafter.

The LS function is a signed distance function to the interface, given by

$$
\phi(\mathbf{x}, t)=\left\{\begin{array}{cl}
\operatorname{dist}(\mathbf{x}, \partial V) & \text { if } \mathbf{x} \in V^{1} \\
0 & \text { if } \mathbf{x} \in \partial V \\
-\operatorname{dist}(\mathbf{x}, \partial V) & \text { if } \mathbf{x} \in V^{2}
\end{array}\right.
$$


where $\partial V$ denotes the interface, $V^{1}$ the region for fluid 1 and $V^{2}$ the region for fluid 2. The LS function is differentiable and facilitates accurate computations for the geometrical properties of the interface.

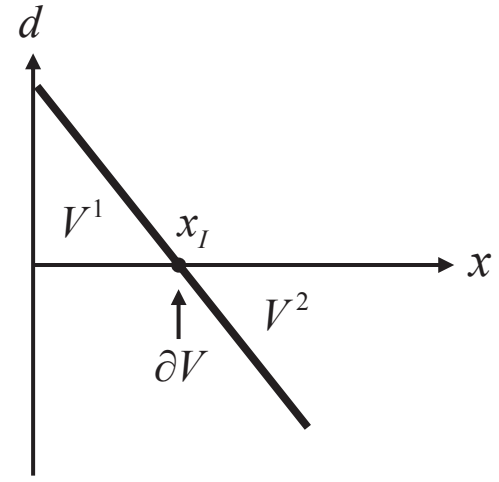

(a)

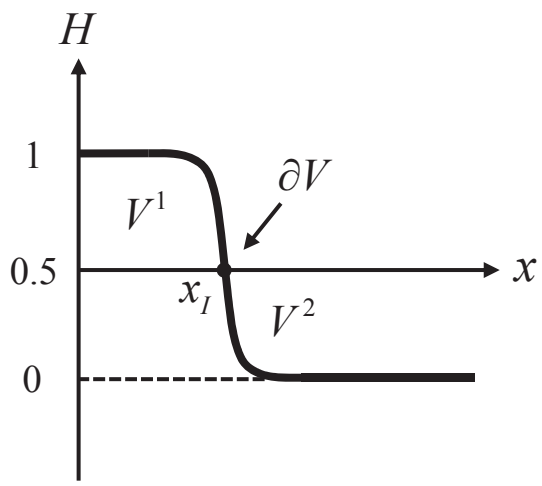

(b)

Figure 1: Schematic diagram of the interface indicator functions in one-dimension: (a) The LS function; (b) The THINC function.

- THINC function (Figure 1(b)):

The THINC function is designed to represent a jump-like solution using the hyperbolic tangent function,

$$
H(\mathbf{x}, t)=\frac{1}{2}(1+\tanh (\beta \phi(\mathbf{x}, t))),
$$

where $\beta$ is a parameter to control the thickness of the interface, i.e. the width of the jump in the hyperbolic tangent function. See a more detailed discussion on $\beta$ in [23].

Being essentially a continuous function, the THINC function is able to approximate the Heavisde step function by increasing the value of $\beta$. The cell-average of THINC function

$$
\bar{H}_{i j k}(t)=\frac{1}{\left|\Omega_{i j k}\right|} \int_{\Omega_{i j k}} H(\mathbf{x}, t) d x d y d z
$$

stands for the volume fraction of a specified fluid in the VOF method. Thus, the rigorous conservation of mass/volume can be automatically guaranteed if the finite volume formulation is applied to solve the THINC function as in the normal VOF schemes.

From the above definitions, the interface can be equivalently identified by either $\phi(\mathbf{x}, t)=0$ or $H(\mathbf{x}, t)=\frac{1}{2}$.

The proposed THINC/LS method updates the LS function $\phi(\mathbf{x}, t)$ and the THINC function $H(\mathbf{x}, t)$ simultaneously and synchronizes them to realize both high-order interface representation and numerical conservativeness. 


\subsection{The evolution equations}

In the present work, we assume that the free interface is passively transported by fluid motion. The advection equation of the LS function $\phi(\mathbf{x}, t)$ reads

$$
\frac{\partial \phi}{\partial t}+\mathbf{u} \cdot \nabla \phi=0
$$

where $\mathbf{u}=(u, v, w)$ is the flow velocity.

The THINC function $H(\mathbf{x}, t)$ follows the same advection equation,

$$
\frac{\partial H}{\partial t}+\mathbf{u} \cdot \nabla H=0
$$

In order to implement the finite volume method, we rewrite (7) into the flux form as

$$
\frac{\partial H}{\partial t}+\nabla \cdot(\mathbf{u} H)-H \nabla \cdot \mathbf{u}=0 .
$$

It is obvious that $(8)$ reduces to

$$
\frac{\partial H}{\partial t}+\nabla \cdot(\mathbf{u} H)=0
$$

for incompressible flows. Shown latter, the cell-average of THINC function $\bar{H}_{i j k}(t)$, i.e the volume fraction or VOF function defined in [5], is updated via the finite volume method so as to ensure rigorous numerical conservation.

\subsection{The THINC/LS method}

As the central part of this paper, we describe the numerical algorithm of the THINC/LS method in this subsection.

We suppose that at time step $n\left(t=t^{n}\right)$, we are given with the VOF values $\bar{H}_{i j k}^{n}$ of the interface cells $\Omega_{i j k}$ where the interface of interest falls in, as well as the values of LS function, $\phi_{i^{\prime} j^{\prime} k^{\prime}}^{n}$, at the centers of the supporting cells $\Omega_{i^{\prime} j^{\prime} k^{\prime}}$, encompassing $\Omega_{i j k}, \Omega_{i j k} \in \cup \Omega_{i^{\prime} j^{\prime} k^{\prime}}$.

\subsubsection{Retrieve the high-order surface polynomial from LS function}

We approximate the cell-wise LS function within cell $\Omega_{i j k}^{n}$ by

$$
\mathcal{P}_{i j k}(x, y, z)=\sum_{s, t, r=0}^{p} a_{s t r} X^{s} Y^{t} Z^{r}
$$

where $\mathcal{P}_{i j k}$ is a polynomial of $p$ th order and $(X, Y, Z)$ is the local coordinates for cell $\Omega_{i j k}$, i.e. $(X, Y, Z)=\left(x-x_{i}, y-\right.$ $\left.y_{j}, z-z_{k}\right) . \mathcal{P}_{i j k}(x, y, z)=0$, referred to as the surface polynomial in our context, represents the 0 -level surface of level set function cutting through the target cell. The coefficients $a_{s t r}$ are computed from the supporting cells $\Omega_{i^{\prime} j^{\prime} k^{\prime}}$ using Lagrange interpolation,

$$
\mathcal{P}_{i j k}\left(x_{i^{\prime}}, y_{j_{l}^{\prime}}, z_{k_{l}^{\prime}}\right)=\phi_{i^{\prime} J_{l}^{\prime} l_{l}^{\prime} k_{l}^{\prime}}^{n}, \quad l \in 0,1, \ldots, p .
$$


Substitute (11) into [10, we get a system of linear equations to find the coefficients $a_{s t r}$. The system in matrixvector form reads

$$
\left[\begin{array}{cccccc}
1 & X_{i_{0}^{\prime}} & Y_{j_{0}^{\prime}} & Z_{k_{0}^{\prime}} & \ldots & X_{i_{0}^{\prime}}^{p} Y_{j_{0}^{\prime}}^{p} Z_{k_{0}^{\prime}}^{p} \\
1 & X_{i_{1}^{\prime}} & Y_{j_{0}^{\prime}} & Z_{k_{0}^{\prime}} & \ldots & X_{i_{1}^{\prime}}^{p} Y_{j_{0}^{\prime}}^{p} Z_{k_{0}^{\prime}}^{p} \\
\vdots & \vdots & \vdots & \vdots & & \vdots \\
1 & X_{i_{p}^{\prime}} & Y_{j_{p}^{\prime}} & Z_{k_{p}^{\prime}} & \ldots & X_{i_{p}^{\prime}}^{p} Y_{j_{p}^{\prime}}^{p} Z_{k_{p}^{\prime}}^{p}
\end{array}\right]\left[\begin{array}{c}
a_{000} \\
a_{100} \\
\vdots \\
a_{p p p}
\end{array}\right]=\left[\begin{array}{c}
\phi_{i_{0}^{\prime} j_{0}^{\prime} k_{0}^{\prime}}^{n} \\
\phi_{i_{1}^{\prime} j_{0}^{\prime} k_{0}^{\prime}}^{n} \\
\vdots \\
\phi_{i_{p}^{\prime} j_{p}^{\prime} k_{p}^{\prime}}^{n}
\end{array}\right]
$$

Considering the symmetry of the supporting cells, polynomials of even order $(p=2,4)$ are considered in this paper.

\subsubsection{Enforce mass/volume conservation from the volume fraction constraint}

In the solution procedure of the present THINC/LS algorithm shown latter, the LS values $\phi_{i j k}^{n}$ are obtained by transporting the LS function with the advection equation, thus the interface defined by $\phi\left(x, y, z, t^{n}\right)=0$ does not guarantee mass/volume conservation. We use the volume fraction $\bar{H}_{i j k}^{n}$ as the constraint condition to enforce the conservation.

Given the surface polynomial $\mathcal{P}_{i j k}$ computed by $\left[10\right.$, as well as the volume fraction $\bar{H}_{i j k}^{n}$, we write the THINC function (4) as,

$$
H(x, y, z, t)=\frac{1}{2}\left(1+\tanh \left(\beta\left(\mathcal{P}_{i j k}(x, y, z)+\phi_{i j k}^{\Delta}\right)\right)\right),
$$

where $\phi_{i j k}^{\Delta}$ is the correction to the level set function due to the volume fraction constraint, which is determined so that

$$
\frac{1}{\left|\Omega_{i j k}\right|} \int_{\Omega_{i j k}} \frac{1}{2}\left(1+\tanh \left(\beta\left(\mathcal{P}_{i j k}(x, y, z)+\phi_{i j k}^{\triangle}\right)\right)\right)=\bar{H}_{i j k}^{n}
$$

is satisfied.

Following [27], we use Gaussian quadrature to approximate the spatial integration in [14], which yields

$$
\sum_{g=1}^{G} w_{g} \frac{1}{2}\left(1+\tanh \left(\beta\left(\mathcal{P}_{i j k}(x, y, z)+\phi_{i j k}^{\Delta}\right)\right)\right)=\bar{H}_{i j k}^{n}
$$

where $w_{g}$ is the normalized weights at Gaussian quadrature points satisfying $\sum_{g=1}^{G} w_{g}=1$. The number of quadrature points should be chosen according to the desired numerical accuracy. See [27] for more discussions. In the present work, we use 3 Gaussian points in each dimension. Newton iteration method can be used to solve 15. Using 0 as the first guess, the iteration converges within a few steps. As long as $\phi_{i j k}^{\Delta}$ is obtained, the interface segment in cell $\Omega_{i j k}$ can be identified as

$$
\mathcal{P}_{i j k}(x, y, z)+\phi_{i j k}^{\Delta}=0
$$

Remarks: we make use of the LS function to find the cell-wise surface polynomial $\mathcal{P}_{i j k}$ that includes all geometrical 
information of the interface. The surface polynomial $\mathcal{P}_{i j k}$ can be viewed as a local LS function for the target cell. However, the interface represented by

$$
\mathcal{P}_{i j k}=0
$$

does not necessarily satisfy the mass/volume conservation. As shown in Figure 2, using the constraint condition (14, we adjust (or synchronize) a distance of $\phi_{i j k}^{\Delta}$ in the normal direction of the interface so as to enforce the conservation.

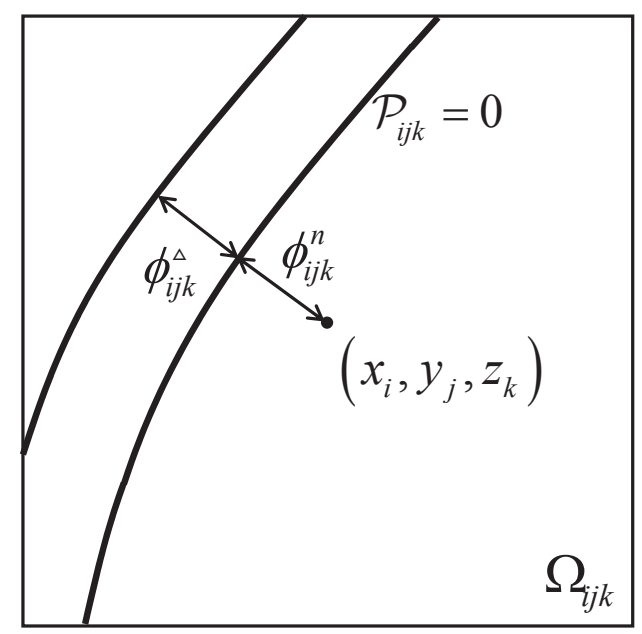

Figure 2: Schematic diagram of the surface polynomial and the volume fraction constraint.

\subsubsection{Reinitialization}

After $\phi^{\Delta}$ are obtained for all interface cells, we need to reinitialize $\phi^{n}$. The reinitialization process is equivalent to solving the Eikonal equation,

$$
|\nabla \phi|=1
$$

with the VOF values $\phi_{i j k}^{n c}=\phi_{i j k}^{n}+\phi_{i j k}^{\Delta}$ fixed in the interface cells, where $\left|\phi_{i j k}^{n c}\right|=\leq \Delta x$.

In the present work, we use the Fast Sweeping Method (FSM) [34] for reinitialization. The LS function after reinitialization $[18], \phi^{n c}$, satisfies the mass/volume conservation condition.

\subsubsection{Update the level set and THINC functions}

Given the mass conserving LS function $\phi^{n c}$ at time step $n\left(t^{n}\right)$, we solve the advection evolution (6) to update the LS function to step $n+1\left(t^{n+1}=t^{n}+\Delta t\right), \phi^{n+1}$. In the present work, we use the fifth-order Hamilton-Jacobi WENO scheme [35] for spatial discretization and the third-order TVD Runge-Kutta scheme [36, 37] for time integration. It it noted that $\phi^{n+1}$ computed from the advection equation does not necessarily satisfy the mass/volume conservation.

The cell-average values of the THINC function, i.e. the volume fractions are updated via the finite volume formu- 
lation as follows

$$
\frac{d \bar{H}_{i j k}(t)}{d t}=-\left(\frac{1}{\Delta x}\left(\mathcal{F}_{i+\frac{1}{2} j k}^{<x>}-\mathcal{F}_{i-\frac{1}{2} j k}^{<x>}\right)+\frac{1}{\Delta y}\left(\mathcal{F}_{i j+\frac{1}{2} k}^{<y>}-\mathcal{F}_{i j-\frac{1}{2} k}^{<y>}\right)+\frac{1}{\Delta z}\left(\mathcal{F}_{i j k+\frac{1}{2}}^{<z>}-\mathcal{F}_{i j k-\frac{1}{2}}^{<z>}\right)\right),
$$

where $\mathcal{F}^{<\alpha>}, \alpha=x, y, z$, denote the numerical fluxes in $x, y, z$ directions respectively, which are computed over each cell surface by Gaussian quadrature. We show the numerical formula for $\mathcal{F}_{i+\frac{1}{2} j k}^{<x}$, the numerical flux in $x$ direction through cell surface segment $S_{i+\frac{1}{2} j k}$, as an example,

$$
\mathcal{F}_{i+\frac{1}{2} j k}^{<x>}=\sum_{g=1}^{G} w_{g}\left(u\left(x_{i+\frac{1}{2}}, y_{g}, z_{g}\right) H_{i^{u p} j k}\left(x_{i+\frac{1}{2}}, y_{g}, z_{g}\right)\right),
$$

where $\left(y_{g}, z_{g}\right)$ stands for the Gaussian points on cell surface $S_{i+\frac{1}{2} j k}$. The subscript $i^{u p}$ indicates the THINC reconstruction function in the upwinding cell which is determined by

$$
i^{u p}= \begin{cases}i, & \text { if } u\left(x_{i+\frac{1}{2}}, y_{g}, z_{g}\right)>0 \\ i+1, & \text { otherwise }\end{cases}
$$

Given the THINC function (13) and the velocity specified at the Gauss points, we get the numerical flux 20] immediately. The same applies to numerical fluxes on other surface segments of $\Omega_{i j k}$.

After the numerical fluxes are obtained, the third-order TVD Runge-Kutta scheme is used to updated to the volume fraction to $n+1$ time step $\left(\bar{H}_{i j k}^{n+1}\right)$.

Note that the THINC reconstruction and the computation to update the volume fraction are limited only to the interface cells identified by $\epsilon \leq \bar{H}_{i j k}^{n} \leq 1-\epsilon . \epsilon$ is a small positive and set to $10^{-8}$ in the present work.

\subsubsection{Summary of the solution procedure}

In order to facilitate the implementation of the present method, we summarize the computation steps to update the function pair from $\left(\phi^{n}, \bar{H}^{n}\right)$ to $\left(\phi^{n+1}, \bar{H}^{n+1}\right)$ as follows.

Step 1: Calculate the surface polynomial $\mathcal{P}_{i j k}$ in 10 for interface cells $\epsilon \leq \bar{H}_{i j k}^{n} \leq 1-\epsilon$ with the coefficients computed from (12) using the values of LS function $\phi^{n}$ in the supporting cells;

Step 2: Compute volume fraction constraint $\phi_{i j k}^{\Delta}$ for interface cells $\epsilon \leq \bar{H}_{i j k}^{n} \leq 1-\epsilon$ that satisfies (14) from the Gaussian quadrature formula 15 with the given volume fractions $\bar{H}^{n}$;

Step 3: Take the interface cells $\left|\phi_{i j k}^{n c}=\phi_{i j k}^{n}+\phi_{i j k}^{\Delta}\right| \leq \Delta x$ as the new 0-level set, and reinitialize the LS values for other cells, which synchronize the level function $\phi_{i j k}^{n}$ to $\phi_{i j k}^{n c}$ to fulfill the mass/volume conservation;

Step 4: Update the level set function from $\phi^{n c}$ to $\phi^{n+1}$ by the Runge-Kutta time integration scheme and finite difference spatial discretization; 
Step 5: Update the volume fraction by (19) where the numerical fluxes of interface cells $\epsilon \leq \bar{H}_{i j k}^{n} \leq 1-\epsilon$ are computed from the THINC function (13) using Gaussian quadrature. The Runge-Kutta is used to predict the volume fraction $\bar{H}^{n+1}$ to new time level.

Step 6: Go back to step 1 to repeat the computations for next time level.

\section{Interface reconstruction tests}

We now assess the interface reconstruction accuracy and convergence rates of the proposed THINC/LS method with some known geometries. In order to quantify the numerical accuracy and convergence rates, we identify the reconstruction error $E_{r}$ as the difference between the area (or volume in 3D) encompassed by the numerical reconstructed interface and the exact interface [9]

$$
E_{r}=\sum_{i j k} \iiint_{\Omega_{i j k}}\left|H(x, y, z)-H^{0}(x, y, z)\right| d x d y d z,
$$

where $H(x, y, z)$ and $H^{0}(x, y, z)$ denote the numerical and exact solutions respectively to the VOF function.

\subsection{Circle reconstruction}

As described in [9], a circle with the radius of 0.368 is centered at $(0.525,0.464)$ in a unit domain. We use the second-order $(p=2)$ and fourth-order $(p=4)$ polynomials to represent the interface. The reconstructed curves are illustrated in Figure 3 . It is fount that both curves coincide well with the exact solution even on a $4 \times 4$ mesh. The fourth-order polynomial gives more accurate results than the second-order one.

For a detailed comparison of different order polynomials, we list the numerical errors and convergence rates in Table 1 and compare them with some classical geometrical VOF methods. As shown in Table 1, the numerical errors and convergence rates of the THINC/LS method are much better than the Piecewise Linear Interface Calculation (PLIC) VOF methods. We observe the high-order convergence rates as expected, which implies that the THINC/LS method can achieve arbitrary order interface reconstruction given the high-order surface polynomial.

\subsection{Sphere reconstruction}

For the three-dimensional case, we conduct the reconstruction test presented in [18]. A sphere is centred at $(0.5,0.5,0.5)$ in a unit cube with radius 0.325 . We use different order $(p=2,4)$ polynomials to reconstruct the surface segments for the interface cells as shown in Figure 4. It is observed that both 2nd and 4th order polynomial surfaces produce accurate results the sphere, and 4th-order polynomial surface coincides better with the exact one.

To compare the THINC/LS method quantitatively against other geometrical VOF methods, we list the reconstruction errors and convergence rates on different mesh resolutions in Table 2. It is observed that the THINC/LS method 


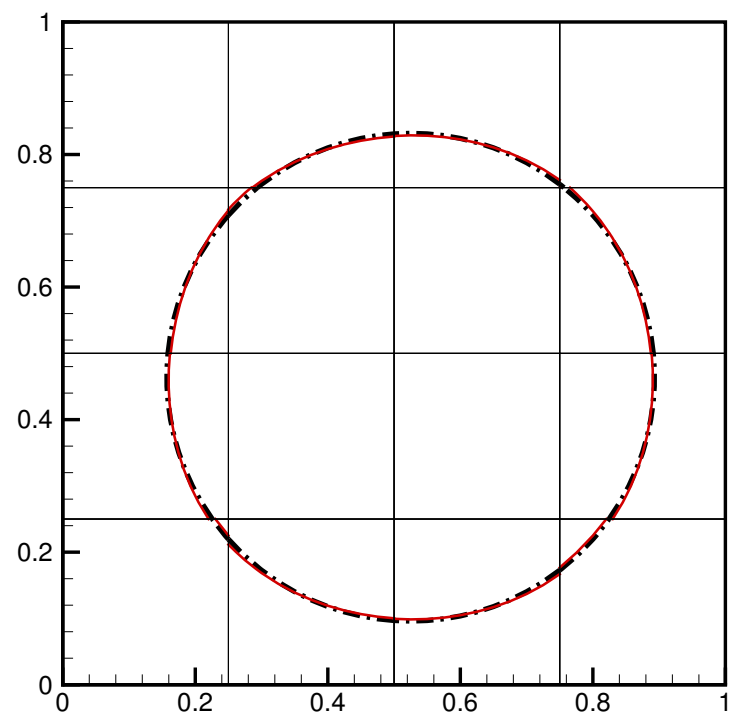

(a)

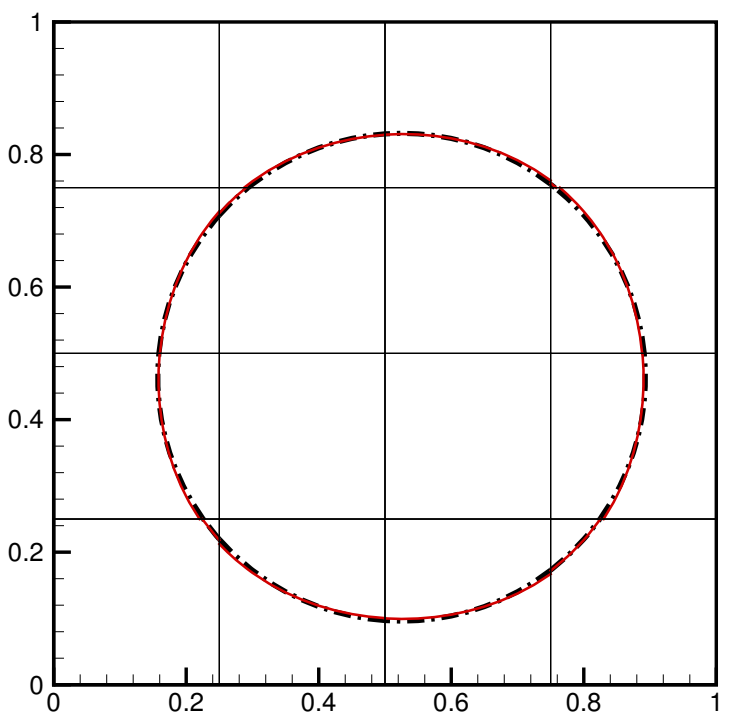

(b)

Figure 3: Reconstructed circle curves using (a) second-order $(p=2)$ and (b) fourth-order $(p=4)$ polynomials on a $4 \times 4$ mesh. Black dash dot line is the exact solution and red solid line is the numerical solution. Both lines are generated of the VOF values sampled at the $10 \times 10$ uniformly distributed sample points within each mesh cell.

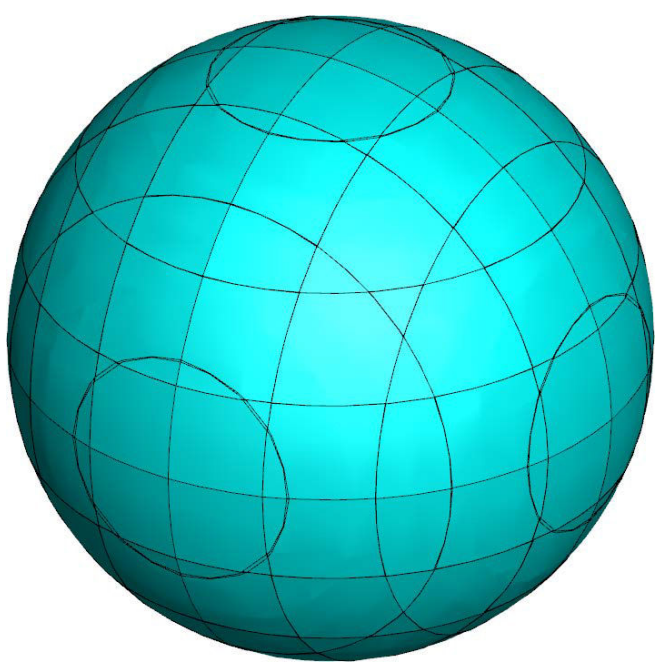

(a)

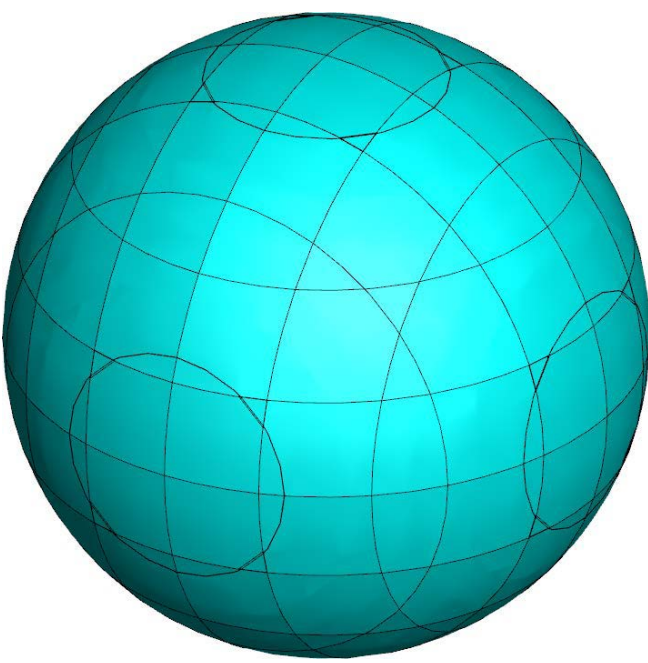

(b)

Figure 4: Reconstructed sphere surfaces using (a) second-order $(p=2)$ and (b) fourth-order $(p=4)$ polynomials on a $10^{3}$ mesh. The surfaces are interpolated from the VOF values at $5^{3}$ uniformly distributed sample points within each cell. The curve lines indicate the edges of the exact and reconstructed surface segments. 

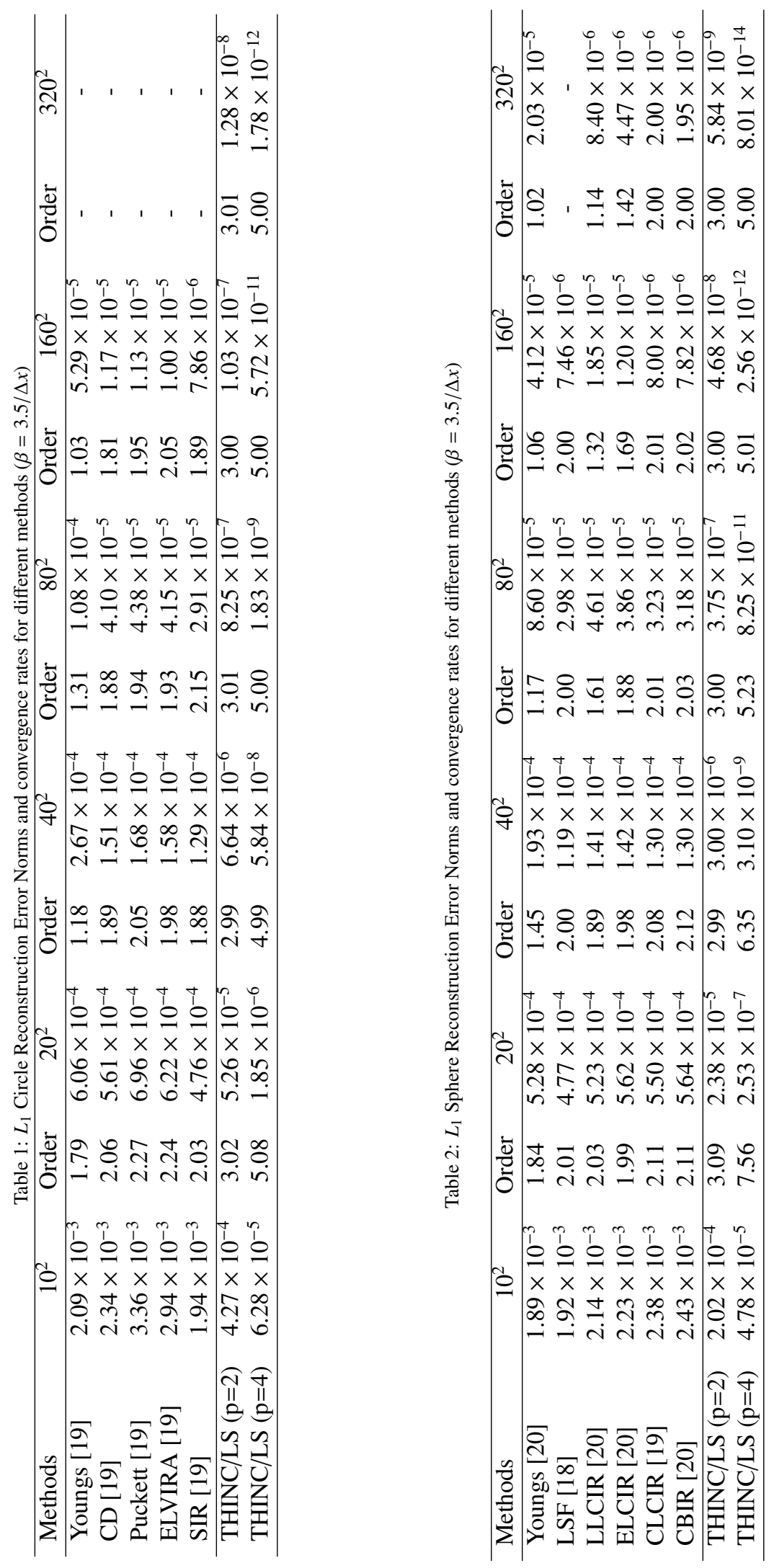
produces more accurate results in than the geometrical VOF methods where low-order surface polynomials were used. It is noted that unlike the geometrical VOF methods which are of significant algorithmic complexity, the interface reconstruction procedure in THINC/LS method simple and straightforwardly extendable to even higher-order in both 2 and 3D.

\section{Advection tests}

In this section, we evaluated the proposed THINC/LS method as an interface-capturing scheme with some widely used advection benchmark tests. To quantify the accuracy of the numerical results, we define the $L_{1}$ error norm for VOF field as

$$
E\left(L_{1}\right)=\sum_{i j k}\left|\bar{H}_{i j k}-\bar{H}_{i j k}^{0}\right|\left|\Omega_{i j k}\right|
$$

where $\bar{H}_{i j k}$ and $\bar{H}_{i j k}^{0}$ stand for the numerical and exact VOF function respectively.

Another widely used measure of the VOF methods is the relative error defined by

$$
E_{r}=\frac{\sum_{i j k}\left|H_{i j k}-H_{i j k}^{0}\right|}{\sum_{i j k} H_{i j k}^{0}} .
$$

\subsection{Translation test}

We firstly conduct the translation test proposed by Rudman [8]. The computational domain $[0,4] \times[0,4]$ is divided into $200 \times 200$ cells. Three types of interfaces are separately translated by a constant velocity field $(2,1)$, which are (a) a hollow square, (b) a tilted $\left(26.57^{\circ}\right)$ hollow square and (c) a hollow circle. The length or radius of the outer and inner interfaces are 0.8 and 0.4 respectively. Both interfaces are initially centered at $(0.8,0.8)$. A CFL number of 0.25 is used in all the computations. As shown in Figure 5 the numerical results of both surface polynomials ( $p=2,4)$ coincide well with the exact solution. The major error of the THINC/LS method comes from the corners of the square, where the right angle is rounded to some extent during the computation. Compared to the 2nd-order surface polynomial, the 4th-order surface polynomial can keep the better-resolved sharp conner.

Quantitative comparisons of the THINC/LS method with other existing VOF methods are given in Table 3 . It is observed that the THINC/LS method can produce more accurate results than the VOF methods especially in the hollow-circle case where high-order surface representation is preferred.

\subsection{Solid body rotation test}

The Zalesak slotted disk test has been widely used to evaluate interface capturing schemes. As described in [39], a slotted circle centered at $(0.5,0.75)$ with radius 0.15 is rotated in a unit domain. After one revolution period, the slotted circle returns back to its initial state if there not any numerical errors. The slot is defined by $(|x-0.5| \leq 0.025$ and $y \leq 0.85)$ and the velocity field is given by $(0.5-y, x-0.5)$. The CFL number in our computation is about 0.25 . 


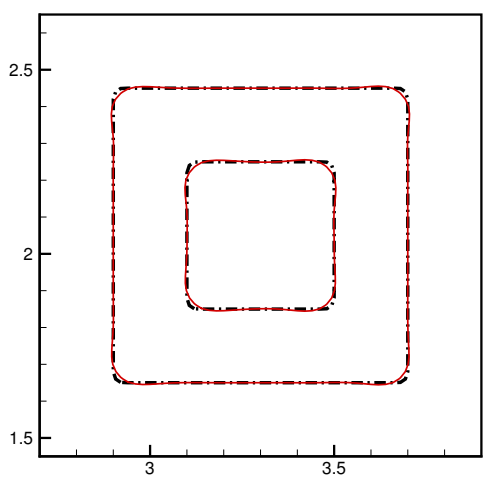

(a)

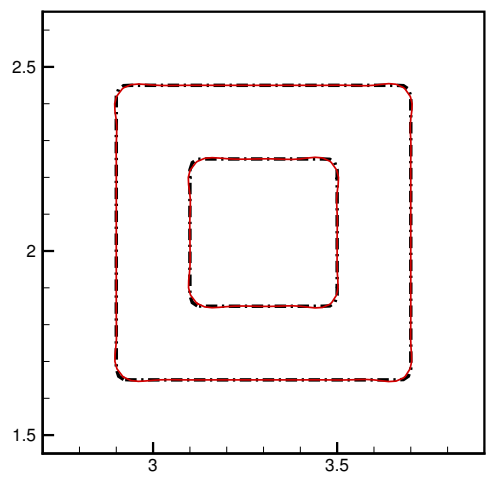

(d)

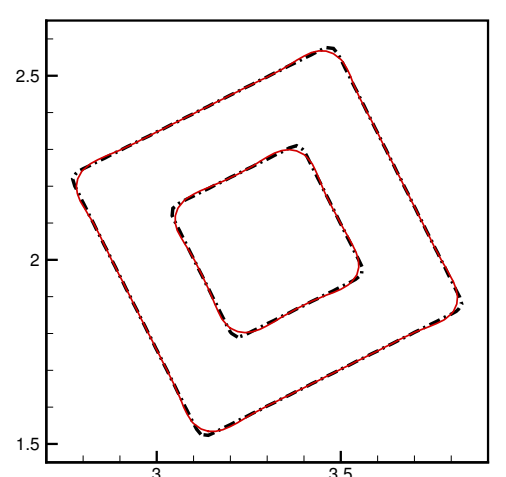

(b)

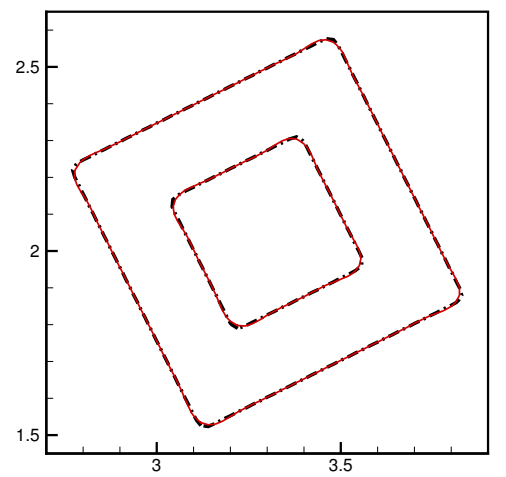

(e)

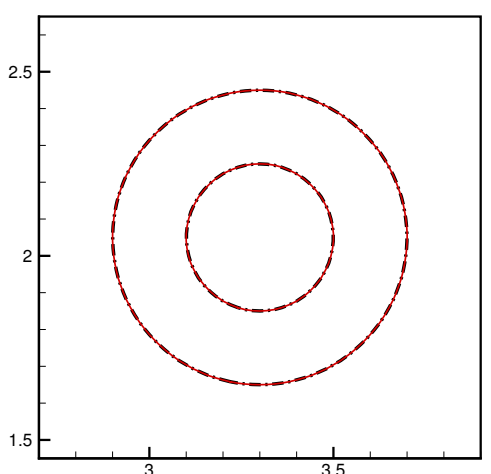

(c)

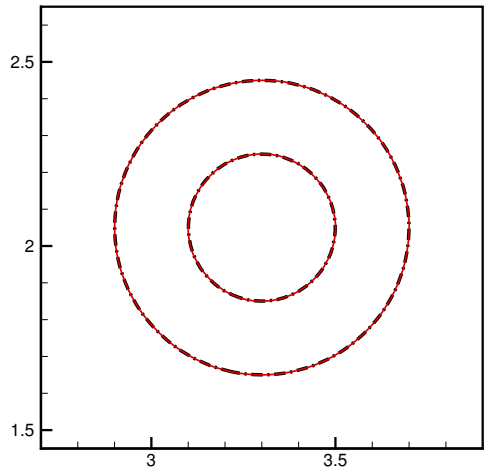

(f)

Figure 5: Numerical results of the Rudman translation test using the second-order (a-c) and fourth-order (d-f) surface polynomials on a $200 \times 200$ mesh. Black dash dot line is the exact solution and red solid line is the numerical solution. Both lines indicate 0.5-contour of the VOF field.

Table 3: Numerical errors $\left(E_{r}\right)$ of different methods in the Rudman translation test $(\beta=3.5 / \Delta x)$

\begin{tabular}{lccc}
\hline Methods & Hollow square $\left(E_{r}\right)$ & Hollow titled square $\left(E_{r}\right)$ & Hollow circle $\left(E_{r}\right)$ \\
\hline SLIC [8] & $1.32 \times 10^{-1}$ & $1.08 \times 10^{-1}$ & $9.18 \times 10^{-2}$ \\
Hirt-Nichols [8] & $6.86 \times 10^{-3}$ & $1.60 \times 10^{-1}$ & $1.90 \times 10^{-1}$ \\
FCT-VOF [8] & $1.63 \times 10^{-8}$ & $8.15 \times 10^{-2}$ & $3.99 \times 10^{-2}$ \\
Youngs [8] & $2.58 \times 10^{-2}$ & $3.16 \times 10^{-2}$ & $2.98 \times 10^{-2}$ \\
Stream/Youngs [38] & $2.70 \times 10^{-2}$ & $3.08 \times 10^{-2}$ & $2.66 \times 10^{-2}$ \\
Stream/Puckett[38] & $3.33 \times 10^{-2}$ & $3.15 \times 10^{-2}$ & $6.96 \times 10^{-3}$ \\
\hline THINC/LS (p=2) & $2.16 \times 10^{-2}$ & $2.24 \times 10^{-2}$ & $2.13 \times 10^{-3}$ \\
THINC/LS $(\mathrm{p}=4)$ & $1.34 \times 10^{-2}$ & $1.22 \times 10^{-2}$ & $2.58 \times 10^{-3}$ \\
\hline
\end{tabular}


Numerical results of THINC/LS method with different orders of the surface polynomials are illustrated in Figure 6 . As Lopez [19] pointed out, the interface reconstruction errors are mainly concentrated at the regions of large interface curvatures, such as the corners of the slot. In most of the VOF methods, the slot of the circle is always found to be distorted after one revolution. However, we don't observe significant distortions of the slot in the results of THINC/LS method that uses high-order interface representations. Moreover, the slotted disk still keeps very well after ten revolutions as shown in Figure 7. It reveals that the THINC/LS method is very geometrically faithful and effective in preserving the shape of the transported interface.

We give the numerical errors after one revolution and compare it with other THINC schemes in Table 4, the THINC/LS method produces superior results than other THINC schemes.

In order to evaluate the computational cost, show the elapse time of the THINC/LS method in comparison with the level set method in Table 5. The THINC/LS method takes nearly twice computational time compared to the level set method, but still within the acceptable range.

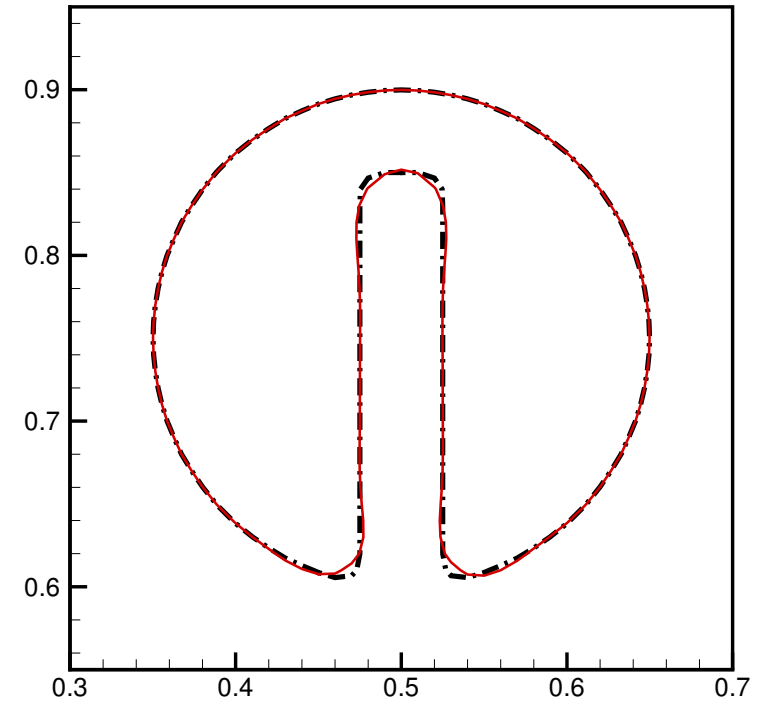

(a)

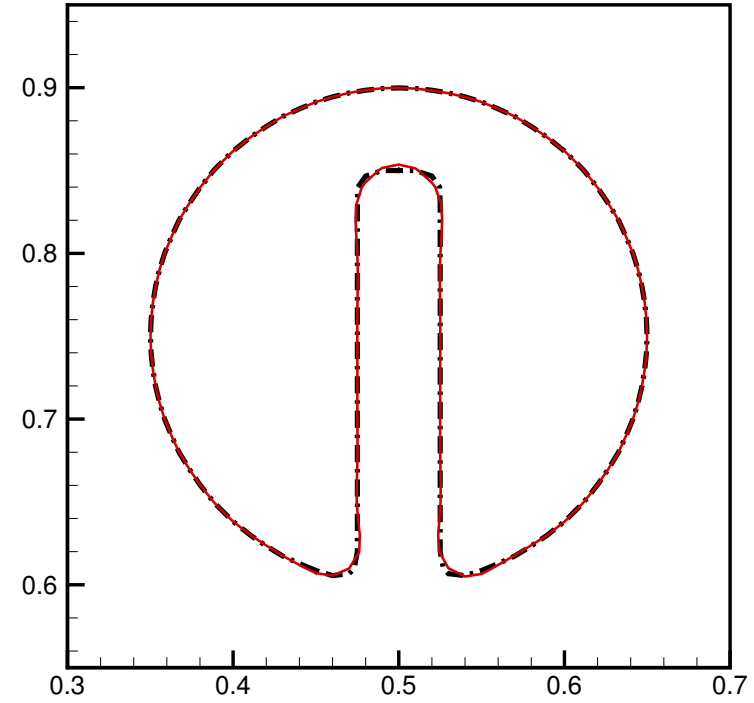

(b)

Figure 6: Numerical results of the Zalesak rotation test after one revolution using (a) second-order and (b) fourth-order (b) surface polynomials on a $100 \times 100$ mesh. Black dash dot line is the exact solution and red solid line is the numerical solution. Both lines are the 0.5 -contours of the VOF fields.

We also conducted the solid rotation benchmark test proposed by Rudman. As described in [8], a slotted circle with radius 0.5 is centered at $(2.0,2.65)$ on a $[0,4] \times[0,4]$ domain. The slot is defined by $(|x-2.0| \leq 0.06$ and $y \leq 2.75)$ and the velocity field is given by $(2.0-y, x-2.0)$. The computations were conducted on a $200 \times 200$ mesh and the maximum CFL number is about 0.25 . Figure 8 shows the numerical results of the THINC/LS method with different order surface polynomials. As discussed before, the main error of the THINC/LS method is concentrated at 


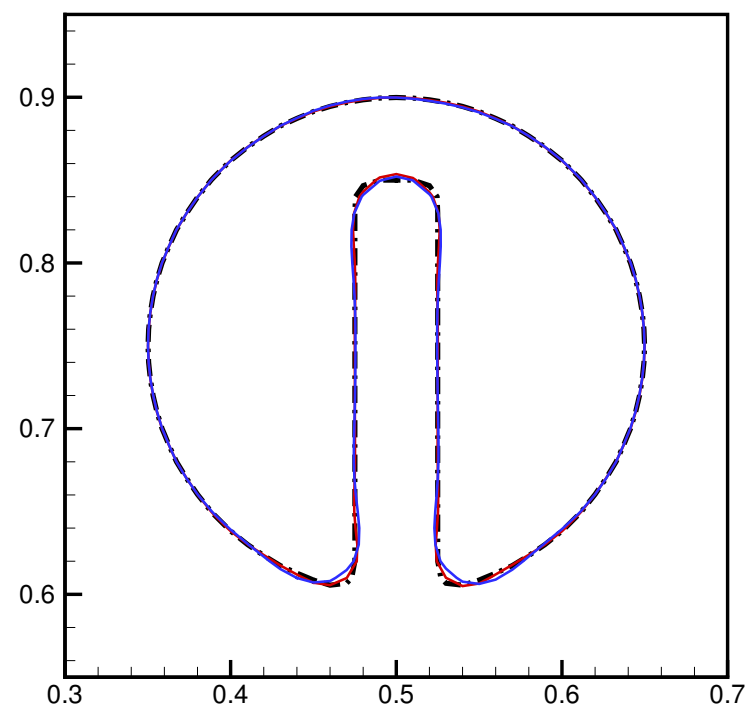

(a)

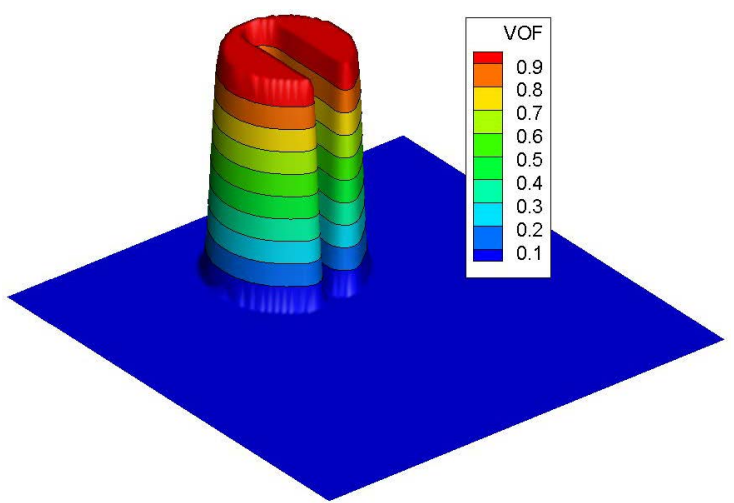

(b)

Figure 7: The 0.5-contour and bird's eye view of the VOF function in the Zalesak rotation test after ten revolution periods using fourth-order surface polynomial on a $100 \times 100$ mesh. In (a), black dash dot line stands for the exact solution, red and blue solid lines are the numerical solutions after one and ten revolutions respectively.

Table 4: Numerical errors $\left(E_{r}\right)$ and convergence rates of Zalesak rotation test after one revolution period $(\beta=3.5 / \Delta x)$

\begin{tabular}{lccccc}
\hline Methods & $50^{2}$ & Order & $100^{2}$ & Order & $200^{2}$ \\
\hline MTHINC [24] & $2.93 \times 10^{-2}$ & 0.86 & $1.61 \times 10^{-2}$ & 1.03 & $7.91 \times 10^{-3}$ \\
UMTHINC [27] & $8.12 \times 10^{-2}$ & 1.63 & $2.61 \times 10^{-2}$ & 0.97 & $1.33 \times 10^{-2}$ \\
THINC/QQ [27] & $8.96 \times 10^{-2}$ & 1.47 & $3.22 \times 10^{-2}$ & 0.95 & $1.67 \times 10^{-2}$ \\
\hline THINC/LS (p=2) & $1.23 \times 10^{-1}$ & 3.04 & $1.50 \times 10^{-2}$ & 1.07 & $7.13 \times 10^{-3}$ \\
THINC/LS (p=4) & $4.83 \times 10^{-2}$ & 2.34 & $9.55 \times 10^{-3}$ & 1.26 & $4.00 \times 10^{-3}$ \\
\hline
\end{tabular}

Table 5: Computation costs of different methods in Zalesak rotation test $(\beta=3.5 / \Delta x)$

\begin{tabular}{lcc}
\hline Methods & One revolution period & Ten revolution period \\
\hline LS (Narrow band) & $12.15 \mathrm{~s}$ & $125.36 \mathrm{~s}$ \\
\hline THINC/LS $(\mathrm{p}=2)$ & $23.01 \mathrm{~s}$ & $237.98 \mathrm{~s}$ \\
THINC/LS $(\mathrm{p}=4)$ & $36.27 \mathrm{~s}$ & $370.11 \mathrm{~s}$ \\
\hline
\end{tabular}


the slot corners. Nevertheless, the slotted circle in general coincides well with the exact solution without significant distortions. To illustrate this, we further compute the numerical errors of the THINC/LS method and compare with other VOF methods. Shown in Table 6 , the results of THINC/LS method are among the most accurate ones.

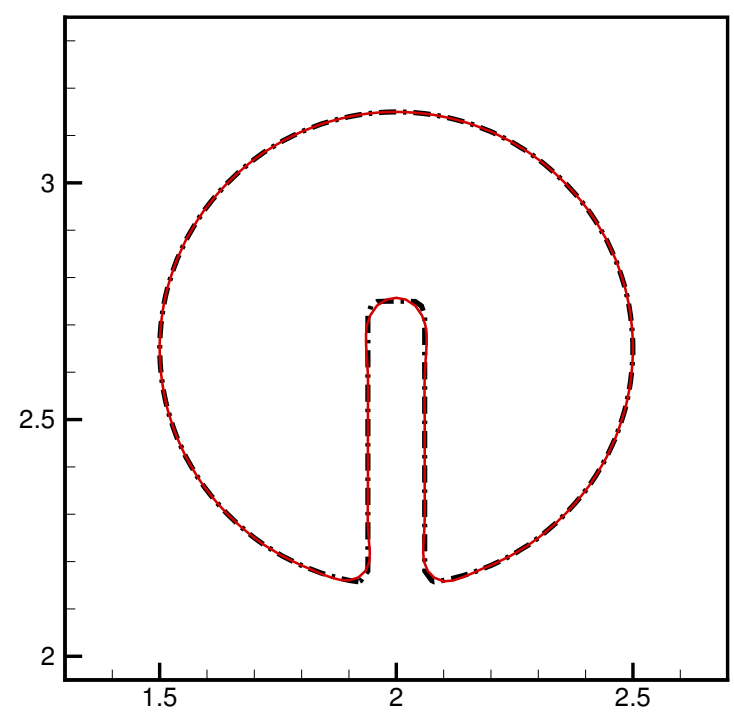

(a)

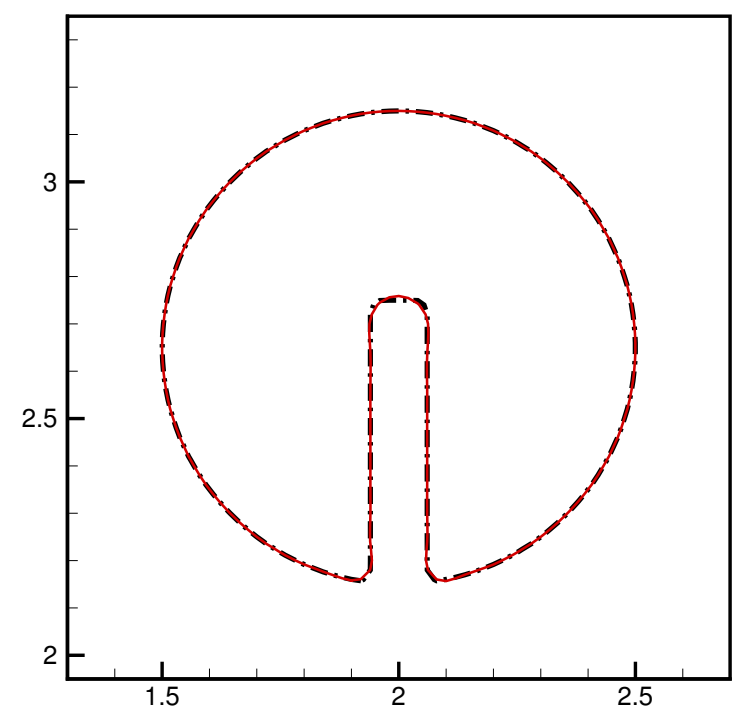

(b)

Figure 8: Numerical results of the Rudman rotation test after one revolution using (a) second-order and (b) fourth-order (b) surface polynomials on a $200 \times 200$ mesh. Black dash dot line is the exact solution and red solid line is the numerical solution. Both lines are the 0.5-contours of the VOF fields.

\subsection{Vortex deformation transport test}

We further assessed the THINC/LS method with the moving interface advected by a deformational velocity field. As described in [9], a circle with radius 0.15 is initially centred at $(0.5,0.75)$ in a unit domain. A time-dependent velocity field is given by the stream function

$$
\Psi(x, y, t)=\frac{1}{\pi} \sin ^{2}(\pi x) \sin ^{2}(\pi y) \cos \left(\frac{\pi t}{T}\right) .
$$

Advected by velocity field (25), the initial circle is firstly distorted and stretched into a spiral shape with a thin tail up to $t=T / 2$, and then transported at a reverse velocity field after $T / 2$ until restoring its initial shape at $T$. For a large $T$, the width of the tail can be thiner the size of mesh cell, and thus not resolvable by the Eulerian fixed grid at $T / 2$. It is well known that the conventional VOF methods tend to generate small droplets or "flotsams" at $t=T / 2$ when the tail of the spiral-like VOF field is stretched into a thin film under grid resolution. In this case, the interface losses its geometrical information, and it is hard to restore the initial state at $t=T$.

We tested the case of $T=8$ on grids with gradually increased resolutions, $32 \times 32,64 \times 64,128 \times 128$. The maximum CFL number of all computations are set to 0.25 . The numerical results on $128 \times 128$ grid at $t=T / 2$ or 
Table 6: Numerical errors $\left(E_{r}\right)$ of the Rudman rotation test $(\beta=3.5 / \Delta x)$

\begin{tabular}{lc}
\hline Methods & Error \\
\hline Hirt-Nichols [5] & $9.62 \times 10^{-2}$ \\
SLIC [14] & $8.38 \times 10^{-2}$ \\
FCT-VOF [8] & $3.29 \times 10^{-2}$ \\
Youngs [8] & $1.09 \times 10^{-2}$ \\
Stream/Youngs [38] & $1.07 \times 10^{-2}$ \\
Stream/Puckett [38] & $1.00 \times 10^{-2}$ \\
DDR/Youngs [40] & $1.56 \times 10^{-2}$ \\
DDR/Puckett [40] & $1.50 \times 10^{-2}$ \\
EMFPA/Youngs [19] & $1.06 \times 10^{-2}$ \\
EMFPA/Puckett [19] & $9.73 \times 10^{-3}$ \\
EMFPA/SIR [19] & $8.74 \times 10^{-3}$ \\
THINC [22] & $3.52 \times 10^{-2}$ \\
THINC/WLIC [41] & $1.96 \times 10^{-2}$ \\
THINC/SW [23] & $1.34 \times 10^{-2}$ \\
THINC/QQ [27] & $1.42 \times 10^{-2}$ \\
Linear fit/Largragian [17] & $9.42 \times 10^{-3}$ \\
Quadratic fit/Lagrangian [17] & $5.47 \times 10^{-3}$ \\
Quadratic fit+continuity/Lagrangian [17] & $4.16 \times 10^{-3}$ \\
\hline THINC/LS (p=2) & $5.09 \times 10^{-3}$ \\
THINC/LS (p=4) & $3.77 \times 10^{-3}$ \\
\hline
\end{tabular}

$t=T$ are shown in Figure 9 where the interface is identified by the 0.5 -contour of the VOF values as the center line of the transition layer. It it observed that THINC/LS method with both 2nd and 4th-order surface polynomials can restore the initial circular shape with adequate accuracy, and the 4th-order polynomial give better solution quality. Without explicit geometrical reconstruction, THINC algorithm exactly conserves the transported field but allows a finite thickness (usually 2 or 3 cell-widths) for the interface transition layer. So, the cells where the VOF values are smaller than 0.5 are not visible by examining only the 0.5 contour. Due to the velocity field $(25)$, the spiral tail is heavily stretched in the flow direction at $T / 2$, which generates VOF values smaller than 0.5 in the surrounding cells. The interface segments in these cell can be identified by $[16$. We plot the reconstructed interface at $t=T / 2$ using (16) in Figure 10 It reveals that the THINC/LS method can reproduce the interface even when the interface is under the grid resolution. Unlike some complex VOF schemes using two non-contiguous linear segments [42] or markers [43] to resolve the thin interfaces, the THINC/LS method is much easer to implement in 3D and unstructured grids. We further show the $L_{1}$ VOF error and convergence rate of the THINC/LS method in comparison with other VOF and hybrid VOF methods in Table 7 It is observed that the THINC/LS method can produce competitive results compared to other hybrid VOF methods.

We also examined the numerical diffusion of the THINC/LS method. Shown in Figure 11, the interface thickness and sharpness maintain satisfactorily after one period $t=T$. 


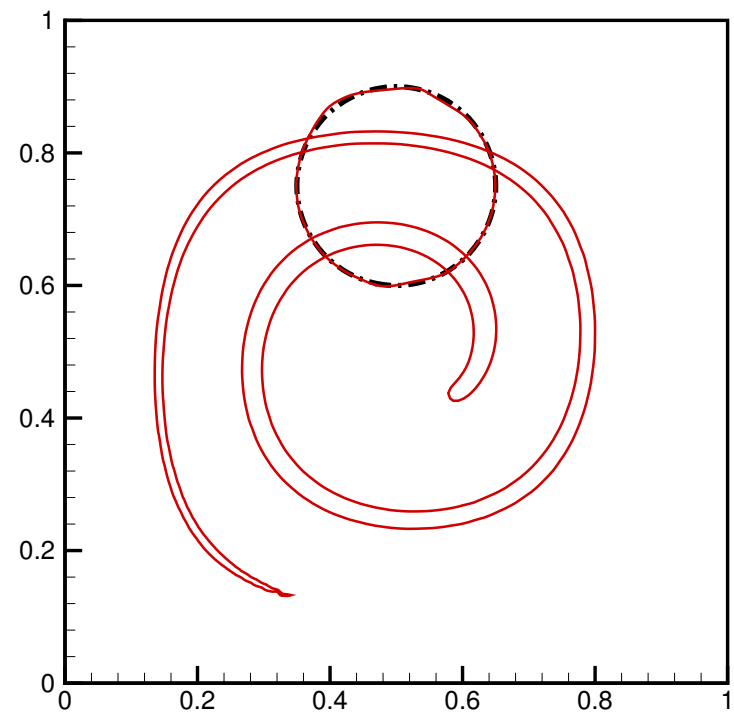

(a)

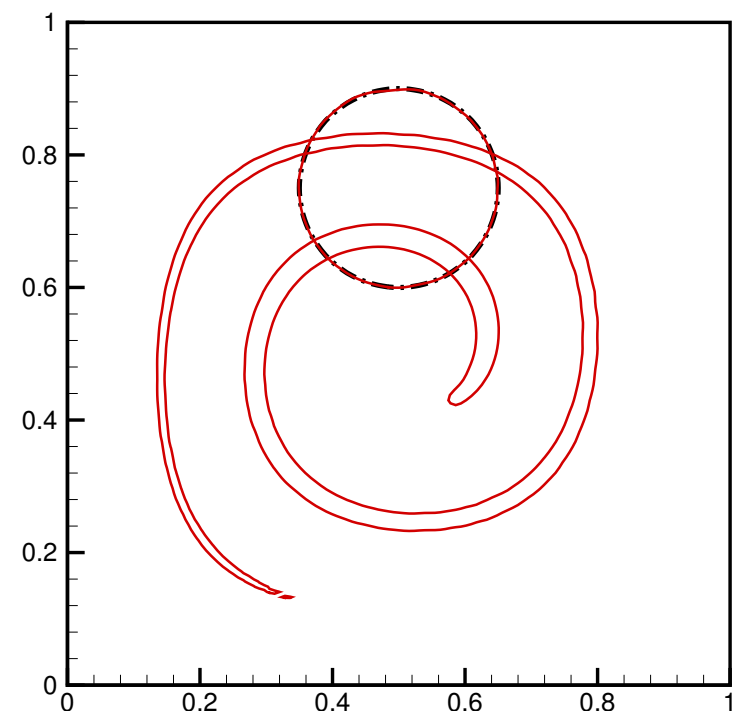

(b)

Figure 9: Numerical results of the Rider-Kothe single vortex test using (a) second-order and (b) fourth-order surface polynomial on a $128 \times 128$ mesh. Solid red line is the numerical results at $t=T / 2, T$ and dashed black line is the exact solution. Both lines are the the 0.5 -contours of the VOF fields.

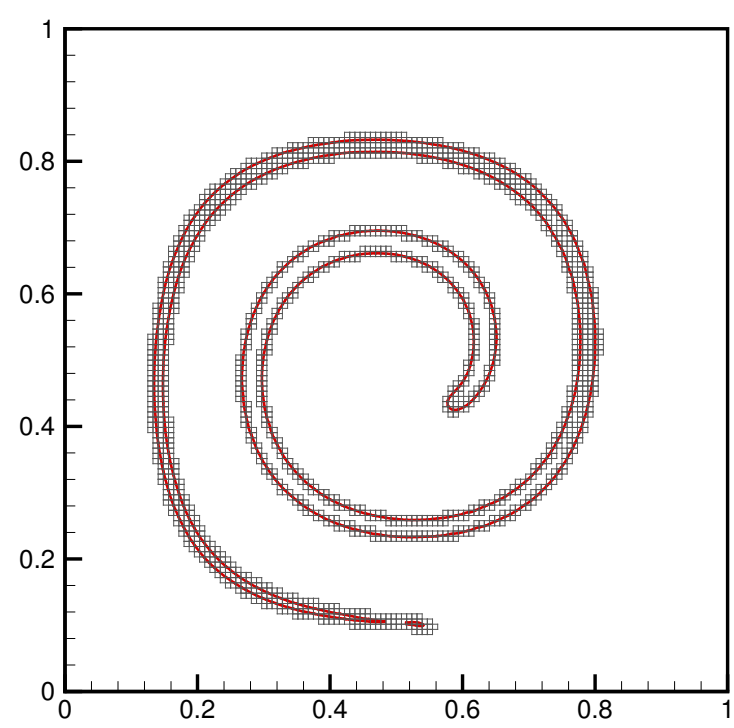

(a)

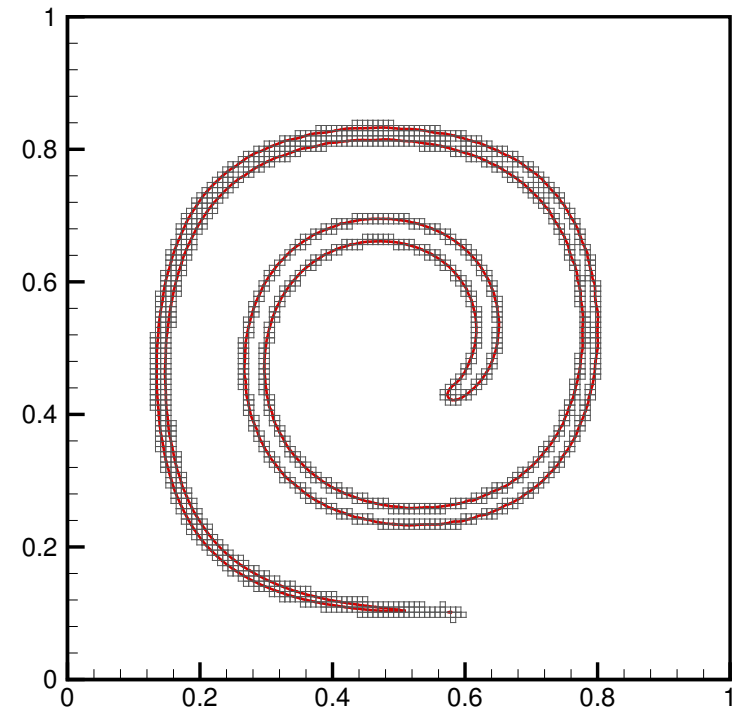

(b)

Figure 10: Reconstructed interfaces of the Rider-Kothe single vortex test using (a) second-order and (b) fourth-order surface polynomials on a $128 \times 128$ mesh 
Table 7: Numerical errors and convergence rates of the time-dependent reversing single vortex test $(\beta=7.0 / \Delta x)$

\begin{tabular}{lccccc}
\hline Methods & $32^{2}$ & Order & $64^{2}$ & Order & $128^{2}$ \\
\hline Rider-Kother/Puckett [9] & $4.78 \times 10^{-2}$ & 2.78 & $6.96 \times 10^{-3}$ & 2.27 & $1.44 \times 10^{-3}$ \\
Stream/Puckett [38] & $3.72 \times 10^{-2}$ & 2.45 & $6.79 \times 10^{-3}$ & 2.52 & $1.18 \times 10^{-3}$ \\
Stream/Youngs [38] & $3.61 \times 10^{-2}$ & 1.85 & $1.00 \times 10^{-2}$ & 2.21 & $2.16 \times 10^{-3}$ \\
EMFPA/Puckett [19] & $3.77 \times 10^{-2}$ & 2.52 & $6.58 \times 10^{-3}$ & 2.62 & $1.07 \times 10^{-3}$ \\
THINC/WLIC [41] & $4.16 \times 10^{-2}$ & 1.37 & $1.61 \times 10^{-2}$ & 2.18 & $3.56 \times 10^{-3}$ \\
THINC/SW [23] & $3.90 \times 10^{-2}$ & 1.36 & $1.52 \times 10^{-2}$ & 1.94 & $3.96 \times 10^{-3}$ \\
THINC/QQ [27] & $6.70 \times 10^{-2}$ & 2.14 & $1.52 \times 10^{-2}$ & 2.31 & $3.06 \times 10^{-3}$ \\
Hybrid markers-VOF [43] & $2.53 \times 10^{-2}$ & 3.19 & $2.78 \times 10^{-3}$ & 2.54 & $4.78 \times 10^{-4}$ \\
Markers-VOF [42] & $7.41 \times 10^{-3}$ & 1.83 & $2.12 \times 10^{-3}$ & 2.31 & $4.27 \times 10^{-4}$ \\
\hline THINC/LS (p=2) & $1.04 \times 10^{-1}$ & 2.66 & $1.65 \times 10^{-2}$ & 3.39 & $1.57 \times 10^{-3}$ \\
THINC/LS (p=4) & $2.92 \times 10^{-2}$ & 2.85 & $4.04 \times 10^{-3}$ & 2.52 & $7.06 \times 10^{-4}$ \\
\hline
\end{tabular}

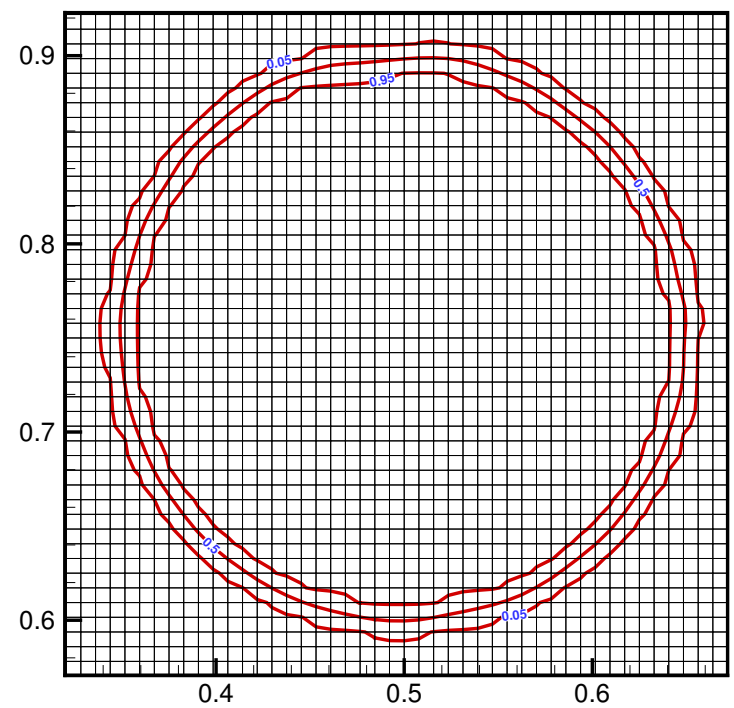

(a)

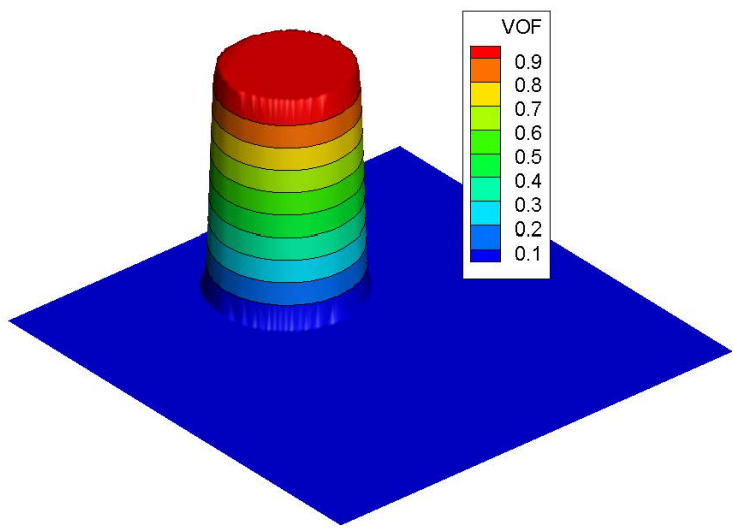

(b)

Figure 11: The $0.05,0.5$ and 0.95 contours and bird's eye view of VOF function in the Rider-Kothe single vortex rotation test after one period $(t=T / 2)$ using the 4-order surface polynomial on a $128 \times 128$ mesh. 


\section{Conclusions}

We propose a novel interface capturing scheme, so-called THINC/LS, for capturing moving interfaces. The innovative and practically-significant aspects of the proposed THINC/LS schemes are summarized as follows:

- Higher-order (arbitrary order in principle) surface polynomials can be determined from the smooth level set function, and then used to build the THINC or VOF function.

- The interface in the level set field is synchronized with the interface identified from the THINC reconstruction with the mass conservation constraint. Thus, the numerical conservativeness is ensured.

- The numerical scheme can produce accurate interfaces even for the interfaces under grid resolution.

- The numerical scheme maintains well the interface thickness and sharpness, as well as the superior geometrical faithfulness of the moving interface.

- The THINC/LS method provides an easy-to-code algorithm for implementation and can be straightforwardly applied to 3D and unstructured grids.

Numerical verifications have been conducted via interface reconstruction tests and advection benchmark tests for moving interfaces evaluate the THINC/LS method. It is revealed that the method can significantly improve the numerical accuracy in comparison with other existing methods through much simpler algorithmic procedure.

\section{Acknowledgments}

The author gratefully thanks to Dr. X. Deng, Dr. B. Xie and P. Jin for their fruitful discussions on this paper. This work was supported in part by the Priority Academic Program Development of Jiangsu Higher Education Institutions (PAPD) and the financial support of the project from the Fundamental Research Funds for the Central Universities NP2016204, as well as the fund from JSPS (Japan Society for the Promotion of Science) under Grant Nos. 15H03916 and $17 \mathrm{~K} 18838$. 


\section{References}

[1] S. O. Unverdi, G. Tryggvason, A front-tracking method for viscous, incompressible,multi-fluid flows, Journal of Computational Physics 100 (1992) 25-37.

[2] G. Tryggvason, B. Bunner, A. Esmaeeli, et al, A Front-Tracking Method for the Computations of Multiphase Flow, Journal of Computational Physics 169 (2001) 708-759.

[3] W. J. Rider, D. B. Kothe, A Marker Particle Method for Interface Tracking, in: 6th International Symposium on Computational Fluid Dyanamics, 1995, pp. 976-981.

[4] W. J. Rider, D. B. Kothe, Stretching and Tearing Interface Tracking Methods, in: 12th AIAA Computational Fluid Dynamics Conference, 1995, pp. 806-817.

[5] C. W. Hirt, B. D. Nichols, Volume of fluid (VOF) method for the dynamics of free boundaries, Journal of Computational Physics 39 (1981) 201-225.

[6] D. L. Youngs, Time-dependent multi-material flow with large fluid distortion, in: Numerical methods for fluid dynamics, Academic Press, New York, 1982, pp. 273-486.

[7] B. Lafaurie, C. Nardone, R. Scardovell, et al, Modeling merging and fragmentation in multiphase flows with SURFER, International Journal for Numerical Methods in Fluids 113 (1994) 134-147.

[8] M. Rudman, Volume-tracking methods for interfacial flow calculations, International Journal for Numerical Methods in Fluids 24 (1997) 671-691.

[9] W. J. Rider, D. B. Kothe, Reconstructing volume tracking, Journal of Computational Physics 141 (1998) $112-152$.

[10] J. E. Pilliod, E. G. Puckett, Second-order accurate volume-of-fluid algorithms for tracking material interfaces, Journal of Computational Physics 199 (2004) 465-502.

[11] S. Osher, J. A. Sethian, Fronts propagating with curvature-dependent speed: Algorithms based on Hamilton-Jacobi formulations, Journal of Computational Physics 79 (1988) 12-49.

[12] S. Osher, R. Fedkiw, Level Set Methods and Dynamic Implicit Surfaces, Springer-Verlag, New York, 2003.

[13] M. Sussman, P. Smereka, S. Osher, A Level Set Approach for Computing Solutions to Incompressible Two-Phase Flow, Journal of Computational Physics 114 (1994) 146-159.

[14] W. F. Noh, P. Woodward, SLIC (Simple Line Interface Calculation), in: Fifth International Conference on Numerical Methods in Fluid Dynamics, 1976, pp. 330-340.

[15] D. L. Youngs, An Interface Tracking Method for a 3D Eulerian Hydrodynamics Code, Technical Report 44/92/35, UK Atomic Weapons Establishment, 1976

[16] E. G. Puckett, A volume-of-fluid interface tracking algorithm with applications to computing shock wave refraction, in: Proceedings of the Fourth International Symposium on Computational Fluid Dynamics, 1991, pp. 933-938.

[17] R. Scardovelli, S. Zaleski, Interface reconstruction with least-square fit and split Eulerian-Lagrangian advection, International Journal for Numerical Methods in Fluids 41 (2003) 251-274.

[18] E. Aulisa, S. Manservisi, R. Scardovelli, S. Zaleski, Interface reconstruction with least-squares fit and split advection in three-dimensional Cartesian geometry, Journal of Computational Physics 225 (2007) 2301-2319.

[19] J. López, J. Hernández, P. Gómez, F. Faura, A volume of fluid method based on multidimensional advection and spline interface reconstruction, Journal of Computational Physics 195 (2004) 718-742.

[20] J. López, C. Zanzi, P. Gómez, et al, A new volume of fluid method in three dimensions-Part II: Piecewise-planar interface reconstruction with cubic-Bézier fit, International Journal for Numerical Methods in Fluids 58 (2008) 923-944.

[21] S. V. Diwakar, S. K. Das, T. Sundararajan, A Quadratic Spline based Interface (QUASI) reconstruction algorithm for accurate tracking of two-phase flows, Journal of Computational Physics 228 (2009) 9107-9130.

[22] F. Xiao, Y. Honma, T. Kono, A simple algebraic interface capturing scheme using hyperbolic tangent function, International Journal for Numerical Methods in Fluids 48 (2005) 1023-1040.

[23] F. Xiao, S. Ii, C. Chen, Revisit to the THINC scheme: A simple algebraic VOF algorithm, Journal of Computational Physics 230 (2011) 7086-7092.

[24] S. Ii, K. Sugiyama, S. Takeuchi, et al, An interface capturing method with a continuous function: The THINC method with multi-dimensional reconstruction, Journal of Computational Physics 231 (2012) 2328-2358.

[25] S. Ii, B. Xie, F. Xiao, An interface capturing method with a continuous function: The THINC method on unstructured triangular and tetrahedral meshes, Journal of Computational Physics 259 (2014) 260-269.

[26] B. Xie, S. Ii, F. Xiao, An efficient and accurate algebraic interface capturing method for unstructured grids in 2 and 3 dimensions: The THINC method with quadratic surface representation, International Journal for Numerical Methods in Fluids 76 (2015) 1025-1042.

[27] B. Xie, F. Xiao, Toward efficient and accurate interface capturing on arbitrary hybrid unstructured grids: The THINC method with quadratic surface representation and Gaussian quadrature, Journal of Computational Physics 349 (2017) 415-440.

[28] J. A. Sethian, Level Set Methods and Fast Marching Methods, Cambridge University Press, Cambridge, 1999.

[29] D. Enright, R. Fedkiw, J. Ferziger, I. Mitchell, A hybrid particle level set method for improved interface capturing, Journal of Computational Physics 183 (2002) 83-116.

[30] M. Sussman, E. G. Puckett, A Coupled Level Set and Volume-of-Fluid Method for Computing 3D and Axisymmetric Incompressible TwoPhase Flows, Journal of Computational Physics 162 (2000) 301-337.

[31] T. Ḿnard, S. Tanguy, A. Berlemont, Coupling level set/VOF/ghost fluid methods: Validation and application to 3D simulation of the primary break-up of a liquid jet, International Journal of Multiphase Flow 33 (2007) 510-524.

[32] X. Yang, A. J. James, J. Lowengrub, et al, An adaptive coupled level-set/volume-of-fluid interface capturing method for unstructured triangular grids, Journal of Computational Physics 217 (2006) 364-394.

[33] D. L. Sun, W. Q. Tao, A coupled volume-of-fluid and level set (VOSET) method for computing incompressible two-phase flows, International Journal of Heat \& Mass Transfer 53 (2010) 645-655. 
[34] H. K. Zhao, A fast sweeping method for Eikonal equations, Mathematics of Computation 74 (2005) 603-627.

[35] G. S. Jiang, D. Peng, Weighted ENO Schemes for Hamilton-Jacobi Equations, SIAM Journal on Scientific Computing 21 (1997) $2126-2143$.

[36] C. W. Shu, S. Osher, Efficient implementation of essentially non-oscillatory shock-capturing schemes, Journal of Computational Physics 83 (1989) 32-78.

[37] S. Gottlieb, On high order strong stability preserving runge-kutta and multi step time discretizations, SIAM Journal on Scientific Computing 25 (2005) 105-128.

[38] D. J. E. Harvie, D. F. Fletcher, A New Volume of Fluid Advection Algorithm: The Stream Scheme, Journal of Computational Physics 162 (2000) 1-32.

[39] S. T. Zalesak, Fully multidimensional flux-corrected transport algorithms for fluids, Journal of Computational Physics 31 (1979) 335-362.

[40] D. J. E. Harvie, D. F. Fletcher, A new volume of fluid advection algorithm: the defined donating region scheme, International Journal for Numerical Methods in Fluids 35 (2001) 151-172.

[41] K. Yokoi, Efficient implementation of THINC scheme: A simple and practical smoothed VOF algorithm, Journal of Computational Physics 226 (2007) 1985-2002.

[42] J. López, J. Hernández, P. Gómez, F. Faura, An improved PLIC-VOF method for tracking thin fluid structures in incompressible two-phase flows, Journal of Computational Physics 208 (2005) 51-74.

[43] E. Aulisa, S. Manservisi, R. Scardovelli, A mixed markers and volume-of-fluid method for the reconstruction and advection of interfaces in two-phase and free-boundary flows, Journal of Computational Physics 188 (2003) 611-639. 